\title{
Research on Defect Detection Technology of Tablets in Aluminum Plastic Package
}

\author{
Yueqiu Jiang*, Shuang Ma, Hongwei Gao and Fei Xia
}

Information Science and Engineering, Shen Yang Ligong University, Shen Yang, China

\begin{abstract}
Aluminum plastic foamed mask package is commonly used for the packaging of tablets in the pharmaceutical companies and defective tablets can be produced during the production of the packaging. This paper studies several key algorithms of defect detection for tablets in aluminum and plastic package and designs the auto- detection software which applies an image processing and recognition technology. Firstly, it uses a weighted filtering method of color images to pretreat the tablet images. Secondly, it classifies the tablets into color and white tablets, and adopts a significant target segmentation method based on the visual significance analysis in order to respectively segment two kinds of tablets from the background image. Then detects the edges of tablets by Canny operator. Finally, it adopts the connected component labeling algorithm to detect the tablets lacking of numbers and other defects including misshapen tablets and tablets with scratches by the SVM algorithm. Large amounts of experiment results demonstrate that the system has greater accuracy and higher stability and speed.
\end{abstract}

Keywords: Color image filtering, connected component labeling, defect detecting, SVM algorithm, visual significance.

\section{INTRODUCTION}

The quality of medicines is very closely tied to its life and health, if defective medicines [1] are supplied into the market, it may not only cause huge damage to people's health, but also will impact the profits of the enterprises in the market competition. The present day packaging of the tablets mainly consist of bottled packages, bagged packages and aluminum plastic foamed mask packages. The aluminum plastic foamed mask packages take a larger share of the packaging market. However, packing tablets need a complicated production process, so some defective tablets are often inevitably produced. With the development of the automated monitoring of the production, a few advanced industrial nations have started using an automatic machine testing method to replace the human labor in pharmaceutical production. Nevertheless, manual detection is still adopted by many nations including China in monitoring the medicine quality, which increases work and has low detection efficiency. As image processing technology, and computer technology rapidly develops, the computer vision also appears to be replacing the human vision. Image process technology is becoming more mature, and computer vision is now used in quality detection of medicine [2]. In china, only a few pharmaceutical companies are trying to use this automated testing system, which shows that the defect detection technology and theory of medicine quality based on image processing is still a bit premature. This paper studies some key questions about tablet defect detection using image processing and machine vision technology. It can bring effective solutions to counter the wastage of human resources and at the same time improve the detection efficiency in medicine packaging, which makes the enterprises constantly create more economic benefits for the society and people.

For defect detection of tablets in aluminum plastic foamed mask package, its task is to automatically recognize three kinds of defects such as lack of numbers on the tablet, misshapen tablets and tablets with scratches on its package. It mainly studies the filtering algorithm of the colored tablet image [3], tablet image segmentation, defect detection of tablets. Color image [4] includes more image information, so weighted filtering algorithm based on color image [5] is used to preprocess the image, which works much better in protecting the details of the image. In tablet image segmentation, the most common segmentation directly used, can't perform well. So it needs to study the classified color and white tablets separately, and extract their different visual significance features to segment the object image based on the information of tablet images. For the tablets lacking numbers, the connected component labeling algorithm is mainly used in defect detection while detection based on BP neural network and support vector machines are used to recognize the misshapen tablets and tablets with scratches. This paper finds that the SVM method used has obvious advantages in accuracy, stability and time.

\section{VECTOR FILTERING ALGORITHM FOR COLOR IMAGES}

Image denoising influences the implementation of the following algorithms including image segmentation and feature extraction, and plays an important role in the image 
processing. Most often, the color images are transformed into gray images to be processed as gray images as this utilizes simple algorithm and faster speed. The pixels of color images are constituted by three components, which means that the color images contain more information that are conducive to the analysis and process the images. In order to preserve the color information, this paper first smooths the image with color image filtering method, which lays a foundation for the following image segmentation.

Vector filtering [6] processes each pixel in the colored image is presented as a 3D vector and each pixel in the image is centered on its filtering window. It computes the aggregated distance from each pixel to all the other pixels in the filtering window, and then the minimum distance is chosen to replace the central pixel of the filtering window. Regarding the computing of the distance using different approaches, three kinds of typical filters are designed [7], they are vector median filter (VMF), basic vector directional filter (BVDF) and directional-distance filter (DDF). They can effectively avoid generating a new color from the pixels in the same way. Assigning different weights to pixels in the filtering window can avoid the above instances, so a kind of weighted vector filter [8] is applied in this paper.

Selection Weighted Vector Filter (SEVF) is a typical weighted vector filter, its output is defined as

$\arg \min \left\{\left[\sum_{m-1}^{N} W_{m} D\left(\mathbf{x}_{k}^{(l)}, \mathbf{x}_{m}^{(l)}\right)\right]^{p} \cdot\left[\sum_{m-1}^{N} W_{m} A\left(\mathbf{x}_{k}^{(l)}, \mathbf{x}_{m}^{(l)}\right)\right]^{1-p}\right\}$

Where the parameter $W_{m}(m=1,2, \cdots, N)$ is a nonnegative real number, and it denotes the weighting coefficient of each pixel in filtering window; $p(0 \leq p \leq 1)$ is the parameter that denotes the importance of module angular distance of a vector in SWVF filter. According to adjusting the parameters $W_{m}$ and $\mathrm{p}$ appropriately, different filters including center-weighted vector median filter (CWVMF) [9] and modified CWVMF (MCWVMF) [10] are generated.

CWVMF:

Each pixel of the filtering window is expressed by the parameter $\mathbf{x}_{k}^{(l)}$, their cumulative distances are given by

$k=1,2, \cdots, N$

Where, $\mathbf{x}^{(l)}\left(\mathbf{x}_{(N+1) / 2}^{(l)}\right)$ denotes the central pixel of the filtering window $\mathbf{W}\left[\mathbf{x}^{(l)}\right]$, the parameter $W_{0}$ is the weight of the central pixel $\mathbf{x}^{(l)}$, and all values of other pixels' weights are 1. $D\left(\mathbf{x}_{k}^{(l)}, \mathbf{x}_{m}^{(l)}\right)$ denotes $L_{2}$ norm of a vector and the vector's formula is $\mathbf{x}_{k}^{(l)}-\mathbf{x}_{m}^{(l)}$.

Arrange all the $d_{k}^{C W}$ of filtering window forming the sequence of pixel vectors in the window, and find the smallest one. Choose its corresponding pixel vector asan output of CWVMF, namely,

$$
\mathbf{y}^{(\text {CWVMF) }}=\arg \min d_{k}^{C W}, k=1,2, \cdots, N
$$

\section{$M C W V M F$ :}

Modify the center-weighted vector median filter (CWVMF) to make the cumulative distance of each pixel in filtering window be given by

$$
d_{k}^{M C W}=\left\{\begin{array}{c}
W_{0} \sum_{m=1}^{N} D\left(\mathbf{x}^{(l)}, \mathbf{x}_{m}^{(l)}\right), k=(N+1) / 2 \\
\sum_{m=1}^{N} D\left(\mathbf{x}_{k}^{(l)}, \mathbf{x}_{m}^{(l)}\right), k \neq(N+1) / 2
\end{array}\right.
$$

Based on the above formula, the output of modified CWVMF(MCWVMF) can be given by

$$
\mathbf{y}^{(\text {MCWVMF })}=\arg \min d_{k}^{M C W}, k=1,2, \cdots, N
$$

Both parameters ${ }_{W_{0}}$ in the filtering algorithms of CWVMF and in MCWVMF must to be optimized, so assign $W_{0}$ in CWVMF and $W_{0}$ in MCWVMF are 4.0 and 5.0 separately. Remove the noise of the image by using five kinds of filters, as shown in Fig. (1).

As shown in Fig. (1a) shows original image with noise, (b)-(d) show that removes the noise by using three typical kinds of vector filters, (b) and (c) show that remove noise by using two kinds of weighted vector filter. The filtering result shows that the typical vector filters performs well in suppressing the noise but introduces blurring artifacts on the edges and detail areas. However, the edge can be well retained at the time of denoising by using weighted vector filters.

\section{TABLET SEGMENTATION IN ALUMINUM PLASTIC FOAMED MASK PACKAGE}

In order to detect and identify the tablets in the aluminum plastic foamed maskpackage, the tablet images need to be segmented after removing the noise, which can separate the tablets from the background. As the experiment finds that,if color images are transformed to gray images directly and if they are segmentedby common methods such as OTSU segmentation and the region grows, then the segmentation perform worse as shown in Fig. (2).

Classify the tablets into color and white tablets based on the color information of tablet and segment them separately. Silver is mostly the color of the background of the tablet plate and its three primary colors have a great differences, however they are similar with white. So the tablets are classified into color and white tablets, and segmented them by using an appropriate method.

\section{A. Distinguish Color Tablets and White Tables}

Judge the tablets on the plates which are white and which are colored, and the step of the color detection are as follow: 


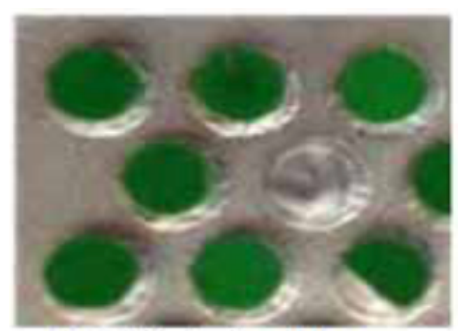

(a) Original image

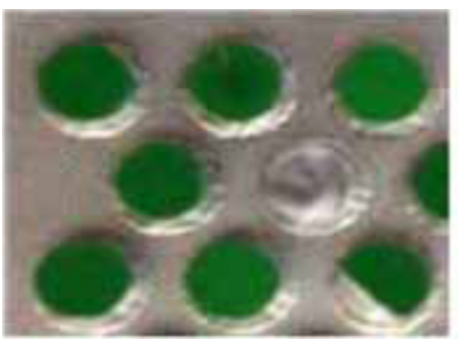

(c) BVDF

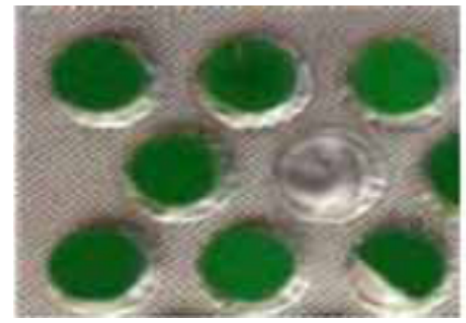

(e) CWVMF

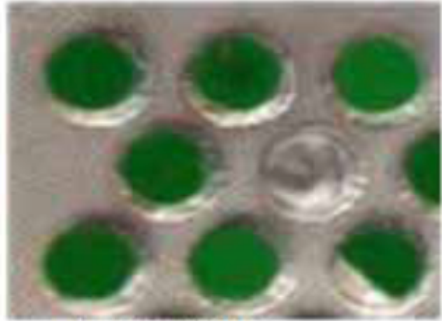

(b) VMF

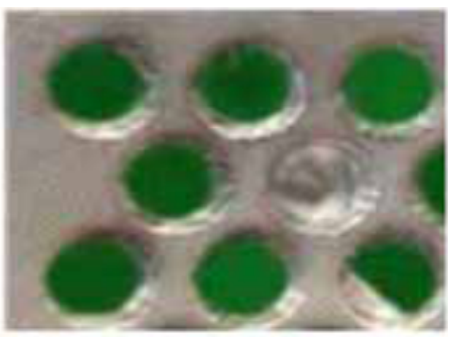

(d) DDF

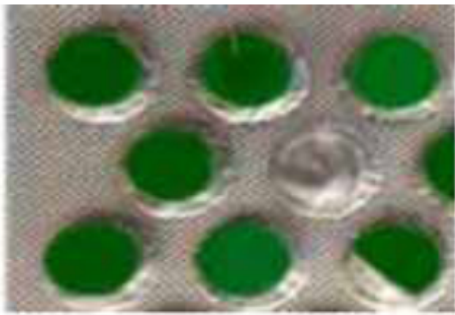

(f) MCWVMF

Fig. (1). Filtering result.

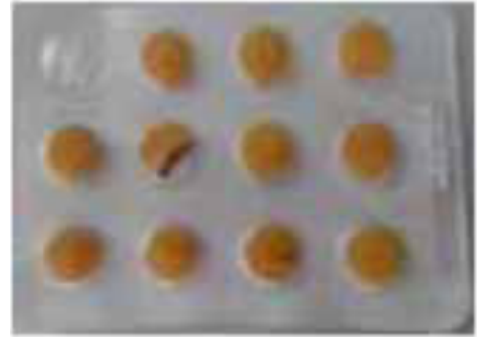

(a) Color Tablets Image

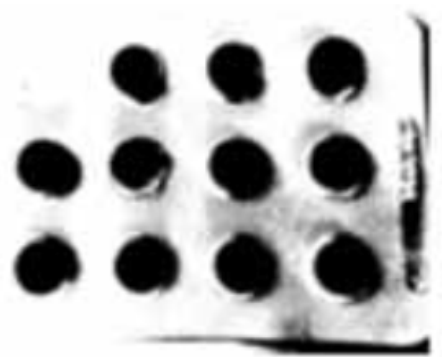

(c) OTSU Segmentation

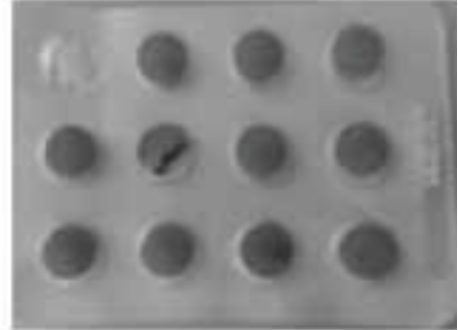

(b) Gray Image

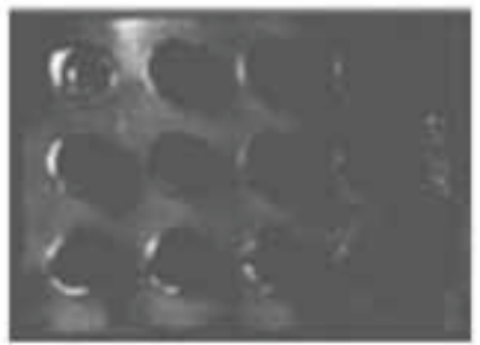

(d) Region Growing

Fig. (2). Two kinds of segmentation. 


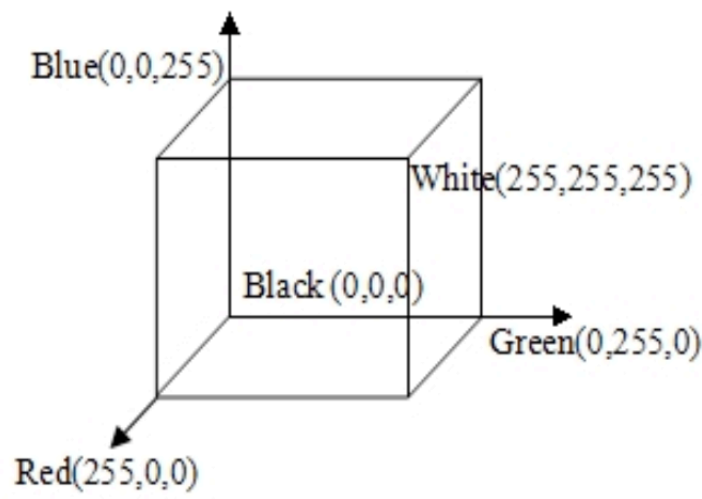

Fig. (3). RGB color space.

Scan the color image, make statistics to the image and count the number of colored pixels [11]. When any difference among the RGB tri-stimulus values of the pixel is greater than $d$, the pixel is judged to be a color pixel and the pixel increases the counter by one.

The total numbers of color pixels that are figured out is denoted by $n$. Then, judge whether the tablets are in color, if $\mathrm{n}>\mathrm{a}$, otherwise judge them to be white. According to large amounts of tests, $\mathrm{d}$ should be 40 and a should be 2000 .

\section{B. Image Segmentation Based on Visual Saliency Atten- tion}

Saliency-based color image segmentation is a popular and efficient segmentation method now. The visual attention mechanism [12] is an significative intrinsic attribute of all animals including humans as well, which makes people ignore the secondary objects and quickly find prominent objects among a mush of information. So it greatly decreases the computational complexity. In saliency-based region detection [13], a variety of features are extracted from the original image, some features are used to recognize the objects and understand the scene under the visual saliency law. The common features in visual saliency detection include spatial luminance, color, orientation, texture, edge feature and phase in frequency domain. So this paper proposes an appropriate feature extraction by visual saliency method to segment the tablet form the background.

\section{Color Tablet Segmentation}

Saliency in a region is closely related to the difference between the features on itself and its surroundings' features. In a saliency region, there is at least one different kind of feature from its neighbors. This paper uses the color property of the image as the feature for color tablets because of its difference between the tablets and the backgrounds. The saliency map is generated by using a global contrast-based approaches [14]. Then it is segmented by OTSU threshold segmentation method, which gets a binary image. In image salient detection, RGB color space and Lab color space that approaches visual sense of human are common color spaces.

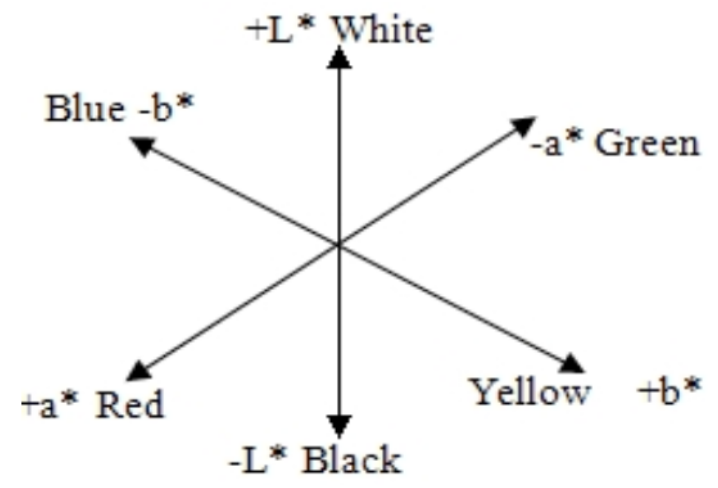

Fig. (4). Lab color space.

All colors of RGB color space in color coordinate can be defined as

$C_{r g b}=a \vec{r}+b \vec{g}+c \vec{b}$

Where, a, b, and c are coefficients of red, green and blue with gray levels in the range $[0,255]$, respectively. There are three channels in Lab space. The first channel is luminance (L), which spans the entire range of possible values, $[0,100]$ and the others are shades, $a$ denotes red and green, $b$ denotes yellow and blue. The schematics of RGB color space and Lab color space are as shown in Fig. (3) and Fig. (4). Lab color space shows a similar visual effect with human, which has some advantages over the RGB color space in this paper.

Local contrast is the difference between the center and its neighborhoods, and the difference near the contour is significant. Global contrast is the difference between the center and the whole image, which separates the object from its surroundings. This paper uses a histogram-based contrast (HC) method 1 [15] to generate saliency maps, smoothens the colors in color space, and segments the saliency map by OTSU threshold segmentation finally. The steps of color tablet segmentation are as follow:

Step 1. Histogram-based contrast method:

HC method computes the saliency based on the difference of pixels' features, which means that the saliency of a pixel is the contrast between this pixel and the others. The saliency of the pixel $I_{k}$ in image $\mathrm{I}$ is defined as:

$S\left(I_{k}\right)=\sum_{I_{i} \in I} D\left(I_{k}, I_{i}\right)$

Where $D\left(I_{k}, I_{i}\right)$ denotes the distance between the pixel $I_{k}$ and the pixel $I_{i}$ in Lab color space. Then formula (7) can be expanded in a pixel order as follows:

$\left(I_{k}\right)=D\left(I_{k}, I_{1}\right)+D\left(I_{k}, I_{2}\right)+\mathrm{L}+D\left(I_{k}, I_{N}\right)$

Where, $\mathrm{N}$ is the number of pixels in image I. Because same-colored pixels also have the same saliency without 

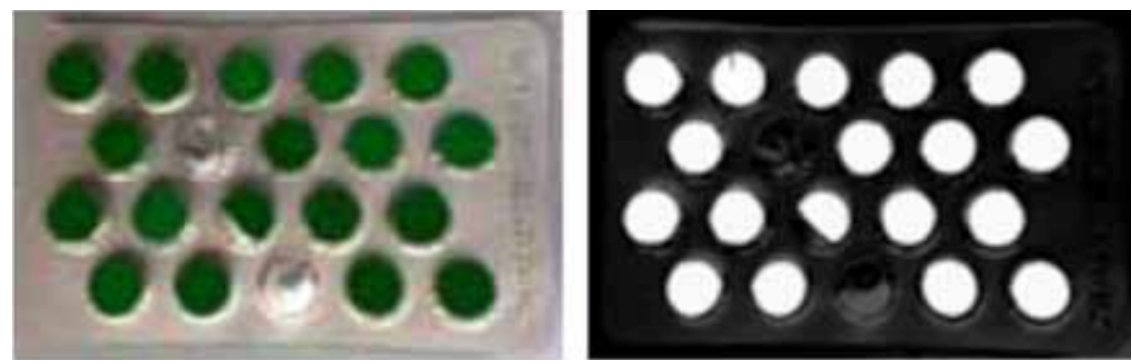

(a) Green Tablets
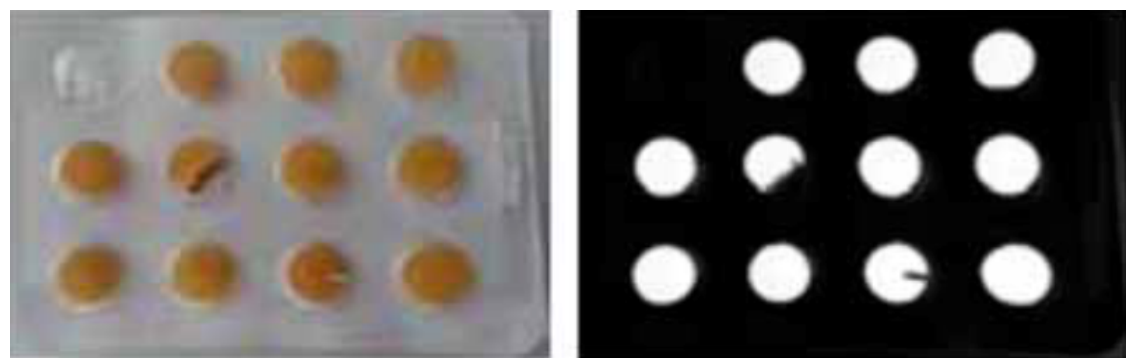

(b) Yellow Tablets

Fig. (5). The saliency map based on HC method.

considering the spatial relationships, tie the same-colored pixels together. So the saliency of each pixel is defined as:

$S\left(I_{k}\right)=S\left(c_{l}\right)=\sum_{j=1}^{n} f_{j} D\left(c_{l}, c_{j}\right)$

Where $c_{l}$ denotes the color of the pixel $I_{k}, \mathrm{n}$ is the number of color pixels in image, $f_{j}$ is the probability of $c_{j}$ occurs in image I.

Step 2. Acceleration based on histogram:

Colors of all channel in RGB space are quantified as twelve colors, which reduces the number of colors to $12^{3}=1728$. This reserve high-frequency colors and makes them be over $95 \%$. The other low-frequency colors should be replaced by the similar colors in the histogram.

Step 3. Smooth in color space:

Color histogram is based on the color quantization and can be used to compute the color contrast, but similar colors may be possibly quantified as different color values. In order to reduce the noise that above process causes, smooth is chosen to improve each color salience value. Namely, each color salience is replaced by the weighted average of its similar color and the salience value. This paper computes the values of color difference in Lab color space instead of RGB space.

The saliency map based on HC detection method is shown in Fig. (5).

Step 4. OTSU threshold segmentation:
In OTSU algorithm, all pixels are divided by the threshold $k$ into two kinds. Pixels whose gray levels are from 0 to $k-1$ belong to the object set $C 0$ while pixels whose gray levels are from $k$ to $L-1$ belong to background set $C 1$. The average gray scale is defined as:

$\mu=\sum_{i=0}^{L-1} i P_{i}$

Where, $P_{i}$ is probability of the ith gray scale. Set $L$ gray scales in image $\mathrm{X}, P_{i}$ is calculated as

$P_{i}=\frac{N_{i}}{N}=\frac{N_{i}}{\sum_{i-0}^{L-1} N_{i}}$

Where, $N$ is the number of all pixels, $N_{i}$ is the number of the $\mathrm{i}^{\text {th }}$-gray pixels in the interval $0 \leq i \leq L-1$.

The percentage of pixels belongs to $C O$ and $C 1$ in total pixels are defined as:

$\omega_{0}=\sum_{i=0}^{k-1} P_{i}$

$\omega_{1}=1-\omega_{0}$

The average gray scale of pixels belong to $\mathrm{CO}$ and $\mathrm{Cl}$ are defined as:

$\mu_{0}=u_{0}(k) / \omega_{0}=\sum_{i-0}^{k-1} i P_{i} / \omega_{0}$

$\mu_{1}=u_{1}(k) / \omega_{1}=\sum_{i-k}^{L-1} i P_{i} / \omega_{1}=\left(1-u_{0}(k)\right) / \omega_{1}$ 


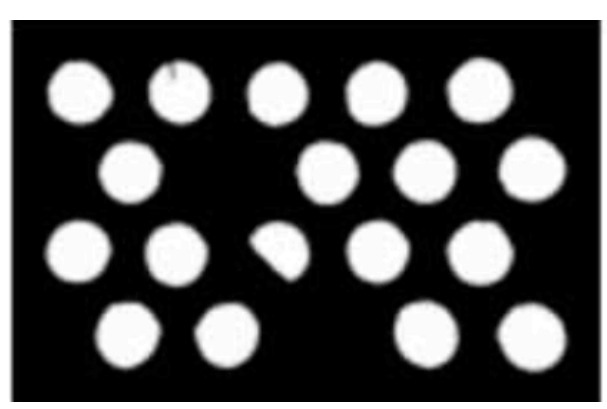

(a) Green Tablets

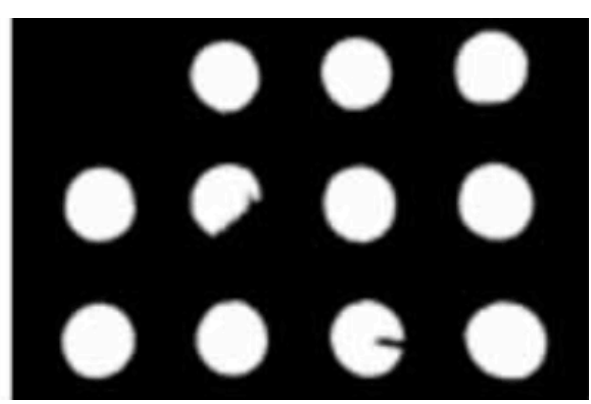

(b) Yellow Tablets

Fig. (6). Effect of color tablets segmented by OTSU.

So the maximum between-cluster variance is defined as:

$\delta^{2}(k)=\omega_{0}\left(\mu-u_{0}\right)^{2}+\omega_{1}\left(\mu-u_{1}\right)^{2}$

Set the difference of $\mathrm{k}$ from 0 to L-1 and compute $\delta^{2}(k)$. The value of $\mathrm{k}$ that makes $\delta^{2}(k)$ a maximum value is the optimal segmentation threshold. The experiment's results demonstrate that, OTSU segmentation performs well and matches the characteristics of human visual system as shown in Fig. (6).

\section{White Tablet Segmentation}

This paper uses texture property as the feature of white tablet image. In texture-image segmentation, Gabor filters are widely used as extractors of local texture information [16]. Gabor filter is actually aGuass function and a multiresolution filter bank that can be generated by varying the orientation and scale of Gabor filter. Filters results in the same filter bank and construct relevant feature vectors, and high dimensional feature can express the texture feature of the image. Naturally, computing the distance of feature vectors can be a texture similarity measure of the two regions in image. For the image $I(x, y)$, Gabor filter image can be defined as:

$\mathrm{I}_{\mathrm{m}, n}(x, y)=\sum_{x_{1}} \sum_{y_{1}} I\left(x_{1}, y_{1}\right) g_{m, n}\left(x-x_{1}, y-y_{1}\right)$

Where $g_{m, n}(x, y)$ is Gabor filter, $m$ is the scale of Gabor filter, $n$ is the orientation of Gabor filter.

The color of white tablets is similar to thesilver background, thus the color tablet segmentation cannot perform well. At first, the white tablet imageis processed by Gabor filter to generate a saliency map, which is also segmented into a binary image by OTSU threshold segmentation, and a morphology erode algorithm is used to process the binary image. Using the center of each region in the resulting image as seed points, the region growing algorithm is adopted to segment the original white tablets image. Eventually, the white tablets are separated from the background successfully, and the binary effects of segmentation are shown in Fig. (7).

\section{TABLET IMAGE EDGE DETECTION}

Comparing different edge detection operators as shown in Fig. (8), using Canny operator to extract the edge of binary image can get better effect. Although Canny edge detection is more complicated, it has a big comparative advantage in position precision and anti-noise property. It is one of the best step edge detection operators, and its effect of detection can be seen in Fig. (9).

\section{DEFECT DETECTION AND RECOGNITION OF TABLETS}

\section{A. Defect Detection by Connected Component Labeling Algorithm}

This paper divides tablet defects into tablets lacking numbers, misshapen tablets and tablets with scratches. Make statistics of the numbers of tablets by the connected component labeling algorithm [17] and judges whether the tablet lacking number exists or not. For a tablet the binary image, the gray value of the background is 0 and the gray value of the object region is set to 1 . Scan the gray image pixel-bypixel first in order to identify the connected pixel regions. When the gray value of scanned pixel is 1 , label it as the object pixel. Examine its four neighbors or eight neighbors to judge whether the object pixels are connected or not. Assume the same label to be connected object pixels and those object pixels whose scanned neighbors are background pixels should be assumed with new labels. After completing the scan, connected background region and some object regions are generated. The number of tablets on the tablet plates is the number of connected regions.

Judge the tablets as being defective if the numbers of tablets gained by connected component labeling algorithm is less than standard numbers. Then send them to the sorting machine in order to fill the vacancy on the tablet plates. Others need be detected whether misshapen tablets or tablets with scratch.

\section{B. Defect Detection and Classification by BP Neural Net Classifier or SVM}

To detect other tablets' defects, first extract the features of the tablet image [18], such as the area, perimeter, circular 


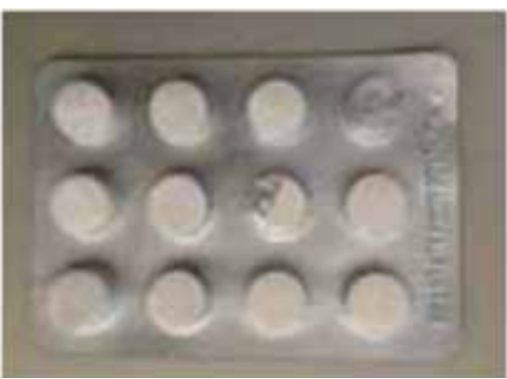

(a) White Tablets Image

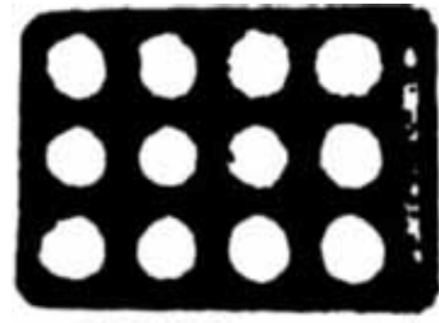

(c) OTSU Segmentation

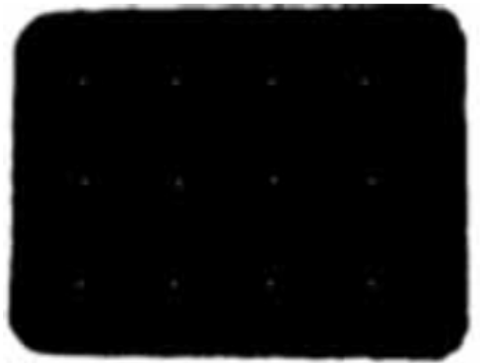

(e) Seed Points

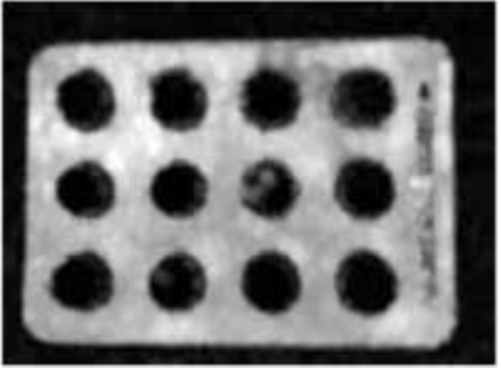

(b) Gabor Saliency Map

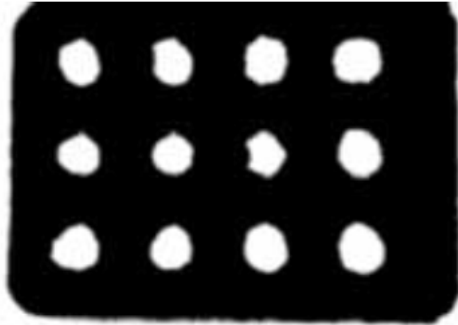

(d) Corrosion Image

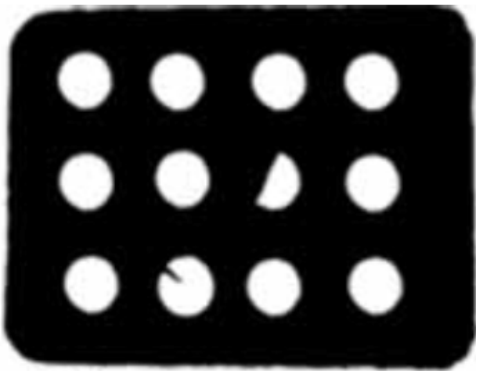

(f) Region Growing

Fig. (7). White tablet segmentation.

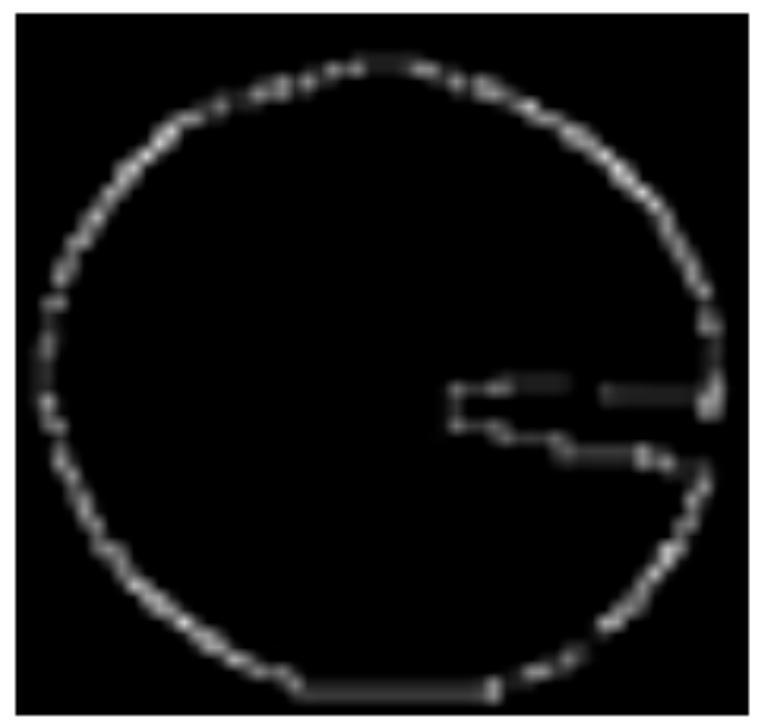

(a) Roberts

Fig. (8). Contd.... 


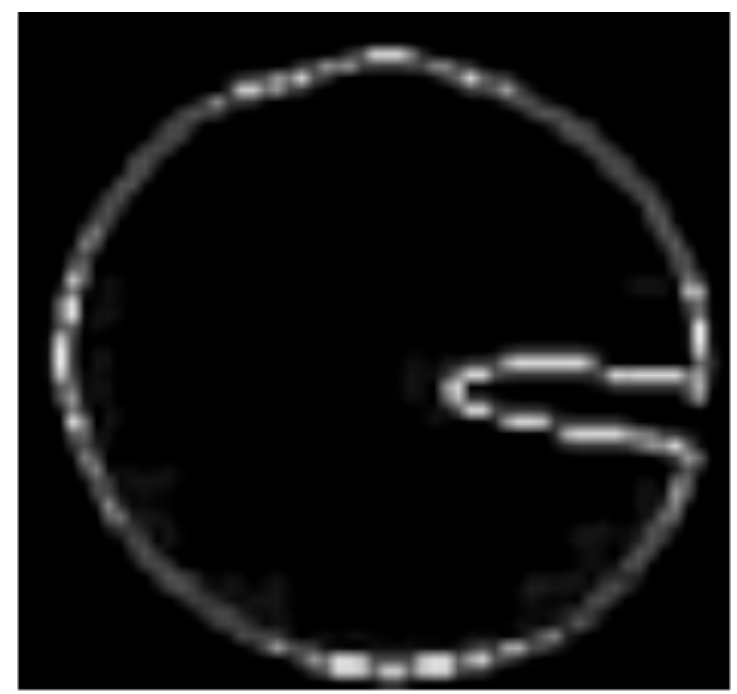

(b) Sobel

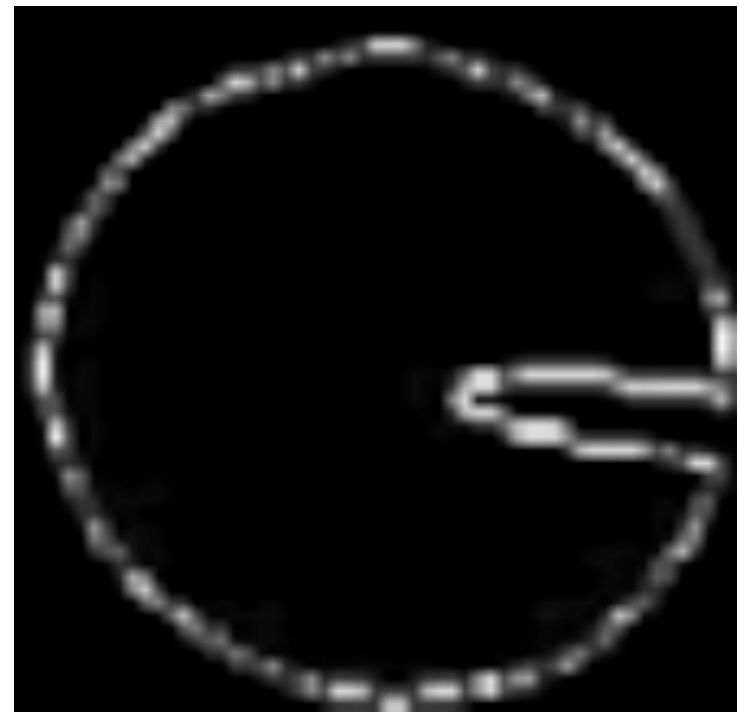

(c) Prewitt

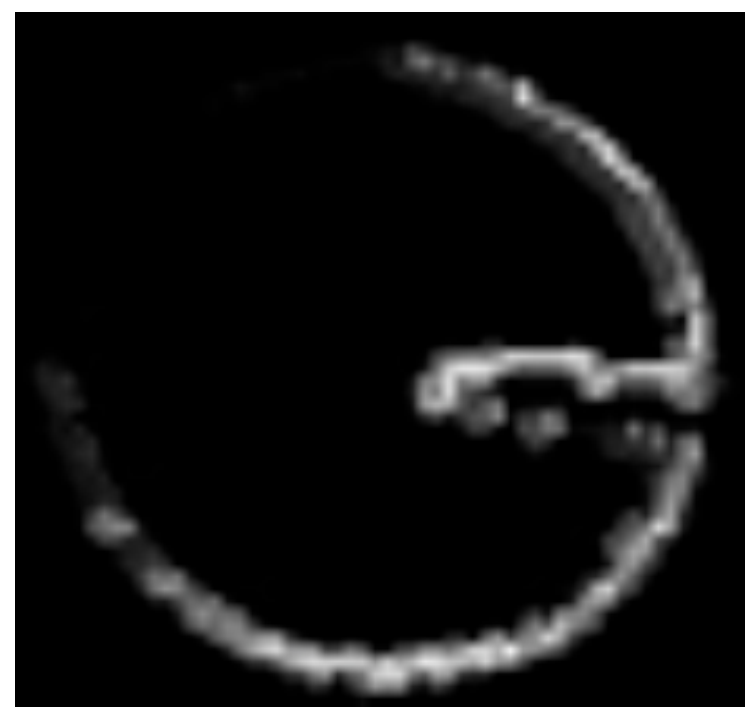

(d) Krisch (Canny Canny)

Fig. (8). Contd.... 


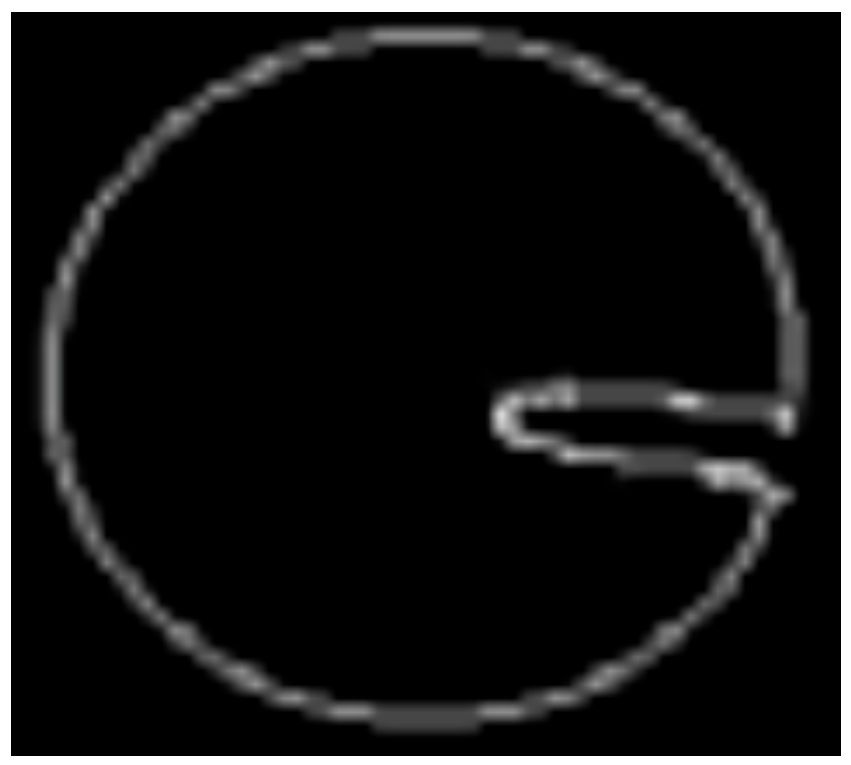

(e) Canny

Fig. (8). Effect of edge detection in different operators.

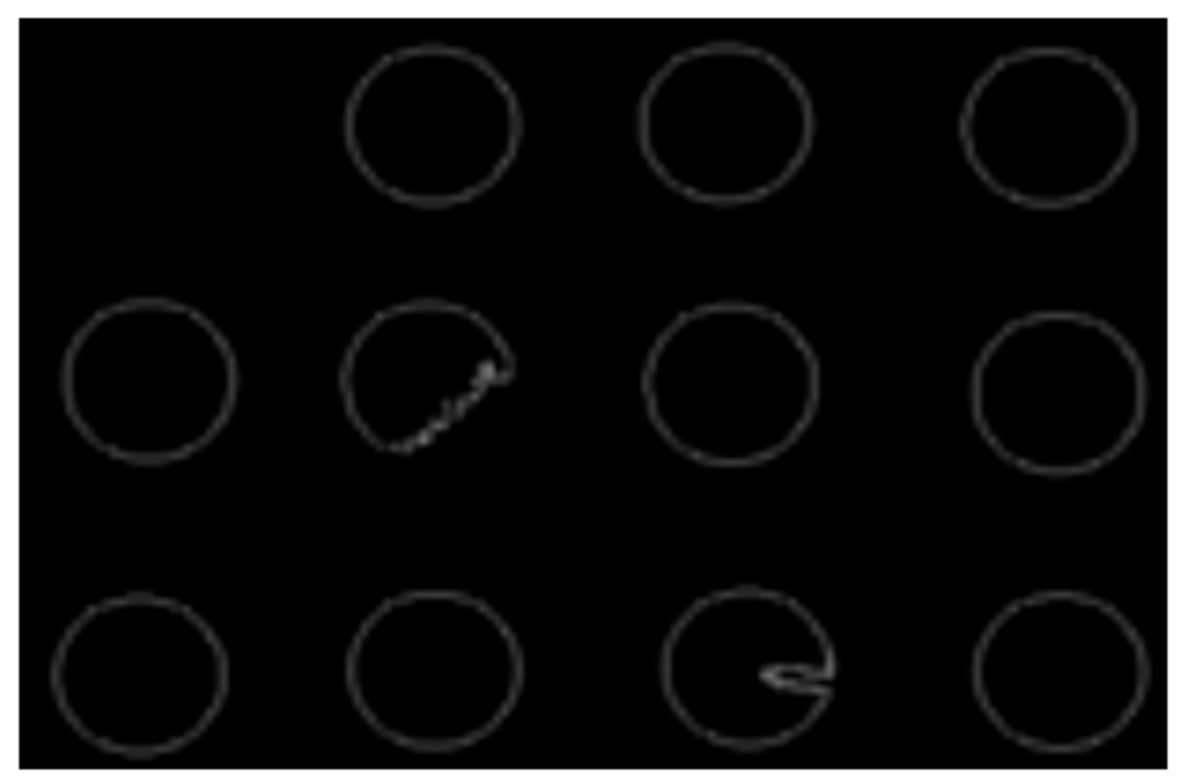

Fig. (9). Effect of edge detection in Canny operator.

ity, the percentage of white pixels in table region and the number of pixels within specific distance from the center of tablets. These five features can judge whether the tablets are qualified.

Neural network theory can be introduced to establish BP neural net classifier based on the feature extraction of tablets defected detection. However, this paper extracts the above five eigenvalues as eigenvectors of the image's edge features and classifies the defects of tablets by using a support vector machine (SVM). The experiment shows that the SVM has better adaptability in small sample event and the steps to establish its identification model are as follow:
Step 1: Select training sample set and deal with the data with normalization.

Step 2: Choose a kernel function and penalty factor $\mathrm{C}$ with high classification accuracy.

Step 3: Shape quadratic optimization problems.

Step 4: Resolve the optimal problem using the optimal method.

Step 5: Substitute the parameter into the kernel unction equation to get SVM model.

120 tablets that belong to two kinds of typical defects are selected as training sample in this paper. 
Table 1. Classification result of Kernel Function.

\begin{tabular}{|c|c|c|c|}
\hline Types of Kernel Function & Liner Kernel Function & Polynomial Kernel Function & RBF Kernel Function \\
\hline \hline Accuracy rate & $83 \%$ & $89 \%$ & $93 \%$ \\
\hline
\end{tabular}

Table 2. Comparison result of optimization parameter in three kinds of method.

\begin{tabular}{|c|c|c|c|}
\hline Optimization Algorithm & Optimal Value $\boldsymbol{O}$ & Optimal Value $\sigma$ & Accuracy Rate \\
\hline \hline Cross validation & 1000 & 0.28 & $96.8 \%$ \\
\hline Genetic algorithm & 1000 & 0.3 & $84.5 \%$ \\
\hline Particle swarm algorithm & 500 & 0.28 & $93.7 \%$ \\
\hline
\end{tabular}

Table 3. Comparison of classification results.

\begin{tabular}{|c|c|c|c|}
\hline Defect Types & Test Sample Size & Accuracy of Recognition Using SVM & Accuracy of Recognition Using BP Network \\
\hline \hline Misshapen tablets & 50 & $97.1 \%$ & $91.2 \%$ \\
\hline Tablets with scratches & 60 & $83.8 \%$ & $75.9 \%$ \\
\hline
\end{tabular}

$\left(x_{1}, y_{1}\right),\left(x_{2}, y_{2}\right), \cdots,\left(x_{n}, y_{n}\right), x_{j} \in \mathbf{R}^{N}$

$y_{j} \in\{1,2\}, j=1,2, \cdots n$

Where $n$ is the number of the samples, that is $120 . N$ is the number of selected eigenvalues and that is 5 .

Test the tablet samples and compare three kinds of kernel function as shown in Table 1. As RBF kernel function can successfully map the samples from a lower-dimensional space of linear inseparability to high-dimensional space of linear separability. The texts show that the method has higher classification accuracy. In order to determine the penalty factor $C$ and the parameter $\sigma$, analysis which method makes it possess a higher classification accuracy in cross validation, genetic algorithm andparticle swarm algorithm. As shown in Table 2, this paperchoses the cross validation and determine that $\mathrm{C}=1000, \sigma=0.28$.

\section{EXPERIMENT RESULTS}

This experiment collects 230 tablet samples with two kinds of defects including nicked tablets and misshapen tablets (the tablet plates are shown in Fig. (10)). 120 samples are used for training including 66 nicked tables and 54 misshapen tablets. The rest of the 110 samples are as test samples including 60 nicked tables and 50 misshapen tables.

Compare the classification results of all samples that are tested by using SVM and BP neural network classifier as shown in Table 3. RBF kernel function is selected and the optimum value of the penalty factor $C$ and the parameter $\sigma$ are determined separately to be 1000 and 0.28 . BP neural network determines the number of nodes of input layer, hidden layer and output layer to be 5-4-2, whereas the number of nodes of input layer corresponds to 5 eigenvalues and the number of nodes of output layer corresponds to 2 kinds of tablet defects.

In cases of small samples, it can be seen that classifying and recognizing the tablet defects using SVM algorithm have higher recognition correct rate and stability than using BP neural network method from the above table. In addition, the SVM algorithm takes 0.09 seconds whereas the BP algorithm takes 0.8 seconds in the test, which means that SVM has lesser speed.

\section{CONCLUSION}

Inspecting defects in during production is more and more important now. This paper carries on a further study on the defect detection technology regarding tablets in aluminum plastic packaging, which includes tablet image filter, tablet image segmentation, feature extraction, defect detection and defect classification of the tablets. After eliminating the noise with weighted filtering method of color image, different appropriate significant targeted segmentation methods based on visual significance are separately used in colored and white tablets. In this paper, BP neural network and SVM methods are separately used for defect detection in multi-sample experiment and it shows that the SVM detect tablets defects faster and more accurately. However, the successful detection of tablets defect still needs improvement in defect types. For example, some minor defects on the face of 


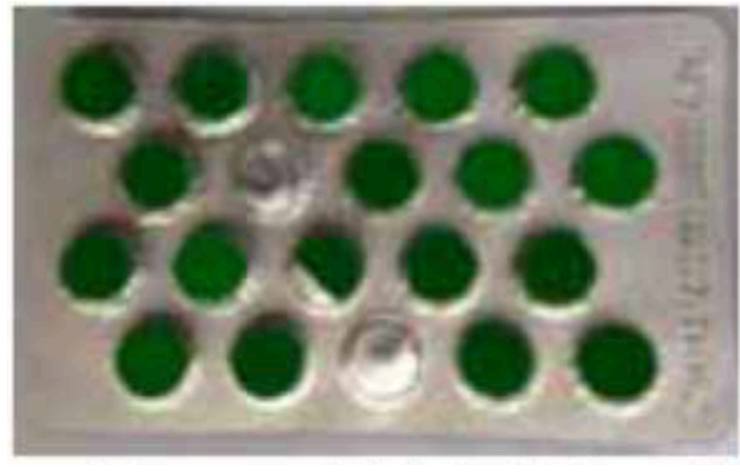

(a) Green Tablet Plates

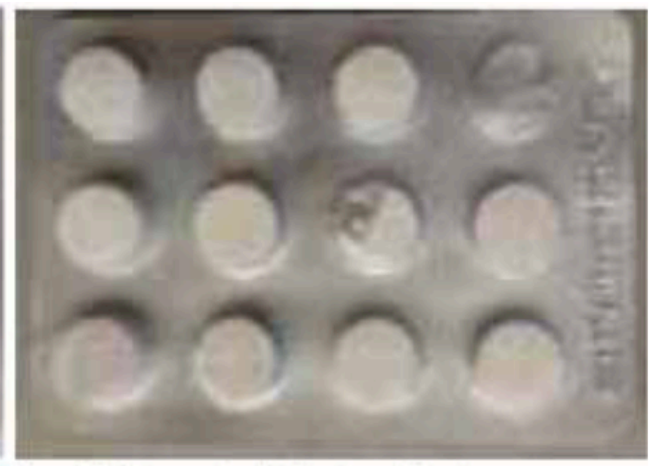

(b) White Tablet Plates

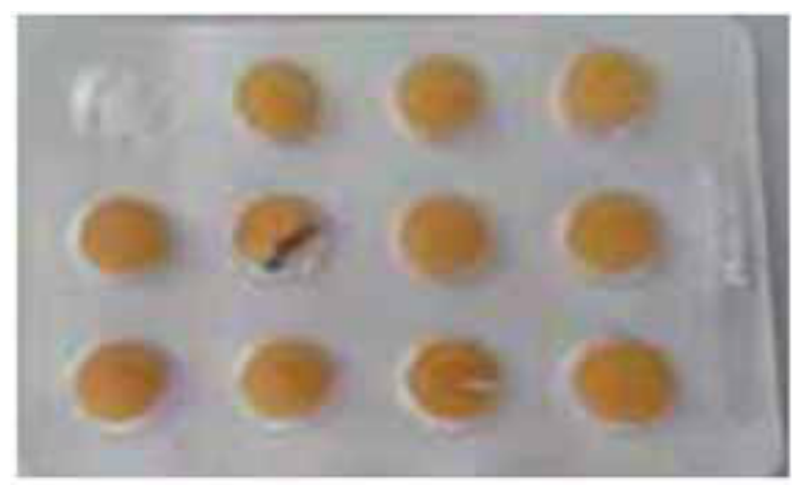

(c) Yellow Tablet Plates

Fig. (10). The defective tablet sample.

tablets, incorrect tablets with different color or some other defects should be detected fast as and far as possible according to theadjustment parameters of the SVM classifier.

\section{CONFLICT OF INTERESTS}

The authors confirm that this article content has no conflict of interest.

\section{ACKNOWLEDGEMENTS}

We would like to express our gratitude thanks to the Communication and Network Institute of Shenyang Ligong University for their invaluable suggestions to improve this paper. This work is supported Liaoning Province second batch of distinguished professor subsidy scheme.

\section{REFERENCES}

[1] M. Song, Y. Zhao, T. Zhao, and P. Ling, "Research on conception of drug defects", Med. Philosophy, vol. 33, no. 9A, pp. 45-47, 2012.

[2] D. Xie, "Research on drug defect inspection in the aluminumplastic blister packaging based on visual inspection technology", Dissertation for the Master Degree of Zhongnan Univ., 2007.

[3] S. Morillas, V. Gregori, and A. Sapena, "Adaptive marginal median filter for colour images", Sensors, vol. 11, no. 3, pp. 3205-3213, 2011.
[4] K. Lin, Y. Wu, and L. Xu, "A survey on color image segmentation techniques," J. Image Graph., vol. 10, no. 1, pp. 1-10, 2005.

[5] Y. Dong and S. Xu, "A new directional weighted median filter for removal of random-valued impulse noise", IEEE Signal Process. Lett., vol. 14, no. 3, pp. 193-196, 2007

[6] L. Jin, X. Yao and D. Li, "A survey on color image vector filtering techniques”, J. Image Graph., vol. 14, no. 2, pp. 243-254, 2009.

[7] J. Xu, L. Wang, and Z. Shi, "A switching weighted vector median filter based on edge detection”, Signal Processing, vol. 98, pp. 359-369, 2014.

[8] L. Jin, and D. Li, "An adaptive spatial distance-weighted vector median filter”, J. Image Graph., vol. 12, no. 6, pp. 970-976, 2007.

[9] B. Smolka, "Efficient modification of the central weighted vector median filter", In: Proc $24^{\text {th }}$ DAGM Symposium Pattern Recog, 2002, pp. 166-173.

[10] B. Smolka, "Modified central weighted vector median filter", $J$. Med. Inform., vol. 3, pp. 41-50, 2002.

[11] X. Jia and B. Ye, "Design of tablet count system based on image processing”, Electron. Des. Eng., vol. 21, no. 12, pp. 67-70, 2013

[12] J. Zhang, L. Shen, and J. Gao, "Regions of interest detection based on visual attention mechanism", Acta Photon. Sin., vol. 38, no. 6, pp. 1561-1565, 2009.

[13] Z. Bo, Z. Lixin, X. Pan, K. Zhou, and Y. Xu, "New approach of fabric defects detection based on saliency region feature", J. Comput. Appl., vol. 32, no. 6, pp. 1574-1577, 2012.

[14] M. M. Cheng, G. X. Zhang, N. J. Mitra, X. Huang and S. M. Hu, "Global contrast based salient region detection", In: IEEE CVPR, 2011, pp. 409-416.

[15] F. Perazzi, P. Krahenbuhl, Y. Pritch, and A. Hornung, "Saliency filters: contrast based filtering for salient region detection", In: IEEE CVPR, 2012, pp. 733-740. 
[16] C. A Basca, and R. Brad, "Texture Segmentation. Gabor Filter Bank Optimization Using Genetic Algorithms", In: IEEE EUROCON, 2007.

[17] S. Cao, "Pills counting algorithm research based on image processing", Dissertation for the Master Degree of Huazhong University of Science and Technology, pp. 31-35, 2011.
[18] S. Liu, "Study on Real-time Tablets image detection and processing system based on image processing and its application", Comput. Mod., no. 5, pp. 66-69, 2013.

Received: November 26, 2014

(C) Jiang et al.; Licensee Bentham Open.

This is an open access article licensed under the terms of the Creative Commons Attribution Non-Commercial License (http://creativecommons.org/licenses/by-nc/3.0/) which permits unrestricted, non-commercial use, distribution and reproduction in any medium, provided the work is properly cited. 